Supplementary Information for

\title{
High spatial resolution imaging of label-free in vivo protein aggregates by VISTA
}

\author{
Li-En Lin ${ }^{\mathrm{a}}$, Kun Miao ${ }^{\mathrm{a}}$, Chenxi Qian ${ }^{\mathrm{a}}$, and Lu Wei *a
}

a. Division of Chemistry and Chemical Engineering, California Institute of

Technology, Pasadena, California 91125, United States

*E-mail: 1wei@caltech.edu 

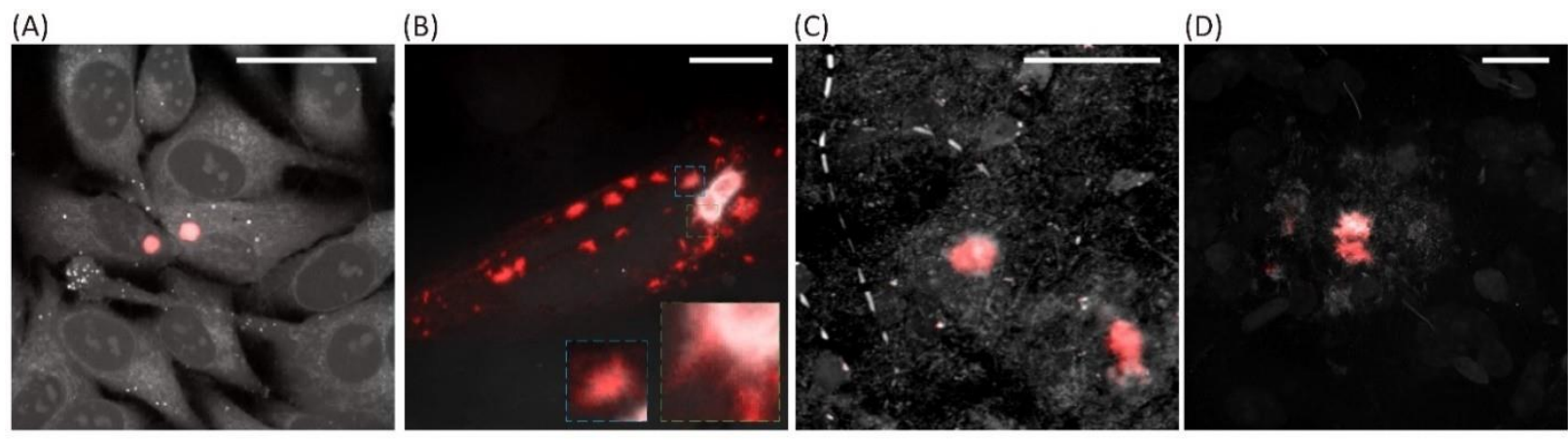

Fig. S1 Merged images of correlative fluorescence (red) and SRS (gray) from Fig. 2. (A) The merged image of Figure 2A (red) and 2B (gray). (B) The merged image of Figure 2C (red) and $2 \mathrm{D}$ (gray). (C) The merged image of Figure 2E (red) and 2F (gray). (D) The merged image of Figure $2 \mathrm{G}$ (red) and $2 \mathrm{H}$ (gray). Scale bars: $40 \mu \mathrm{m}$.
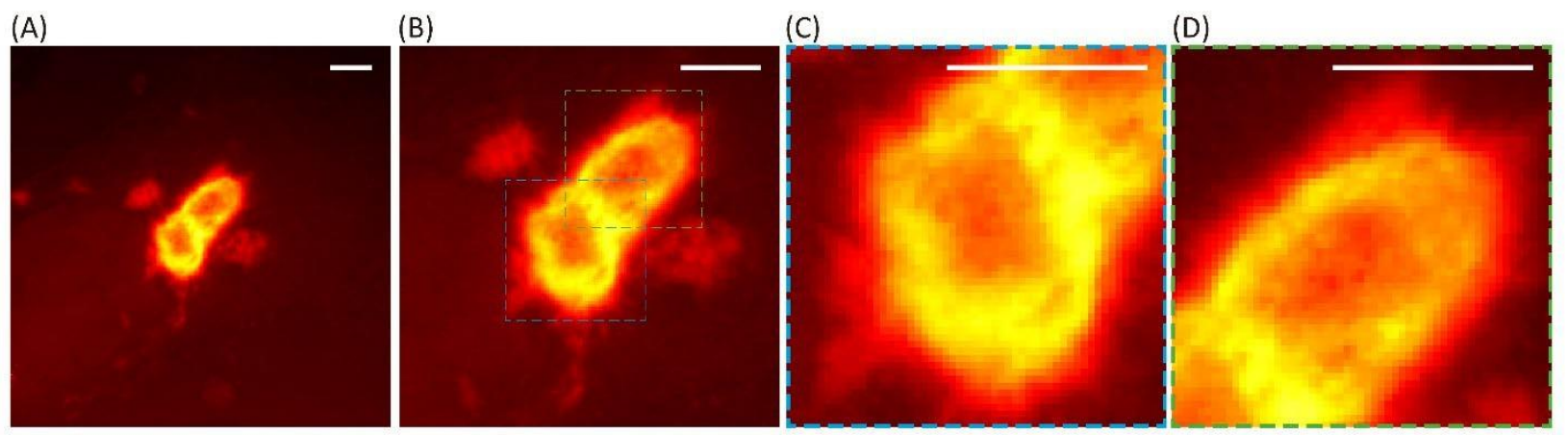

Fig. S2 Internal hollow structures for the PolyQ aggregates shown in Fig. 2B with adjusted image contrast. (A) VISTA image of the PolyQ aggregates in Fig. 2B with a larger intensity contrast range. (B) Zoomed in view from (A) for the same aggregate region. (C-D) Zoomed in views in (B) from the dashed boxes. Blue dashed box is shown in (C) and green dashed box is shown in (D). scale bars: 10 $\mu \mathrm{m}$. 
(A)

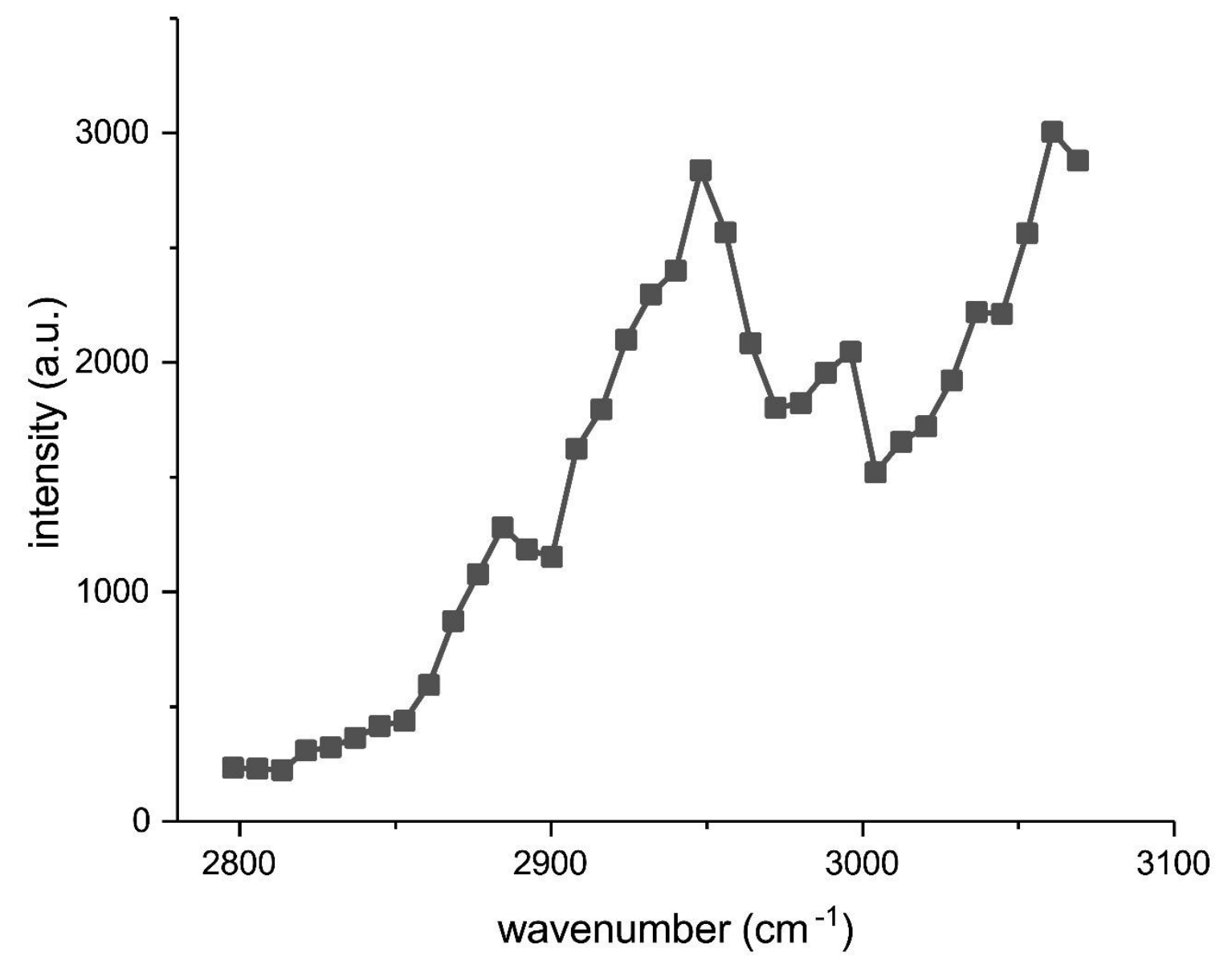

(B) Before gelation

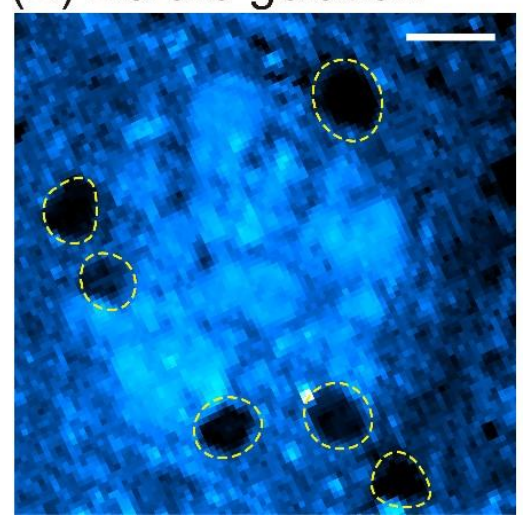

(C) Expanded in water

(D) Overlap
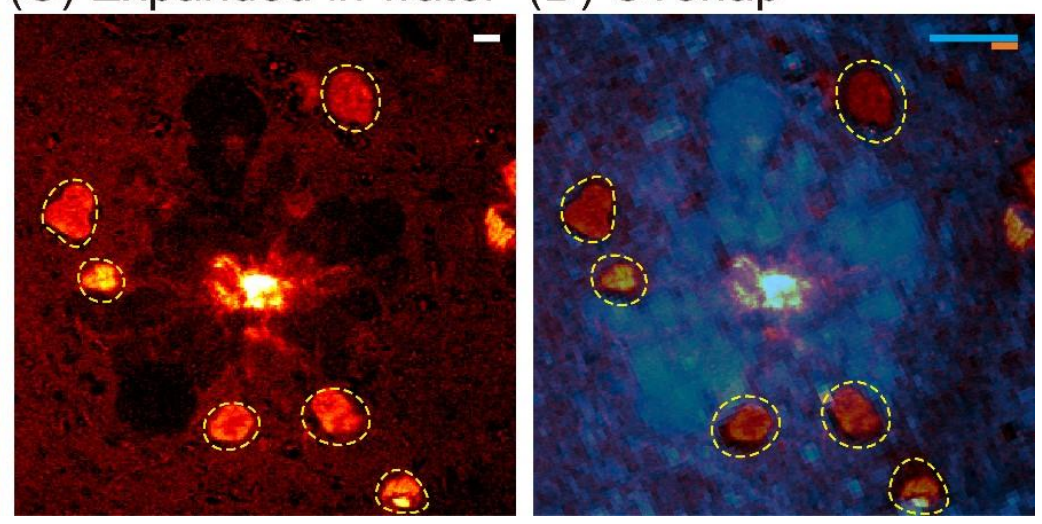

Fig S3. (A) The hSRS spectra of the expanded $\mathrm{A} \beta$ plaques in the $\mathrm{CH}$ region with a diminished spectral feature of lipid peaks around $2840 \mathrm{~cm}^{-1}$. (B-D) Registration of the same field of views before and after expansion from the same plaque-containing brain tissue samples. (B) Before gelation, (C) expanded in water, showing the clear removal of lipids, and (D) overlap of B\&C. The nuclei are circled by yellow dashed lines. Scale bars: $10 \mu \mathrm{m}$. 

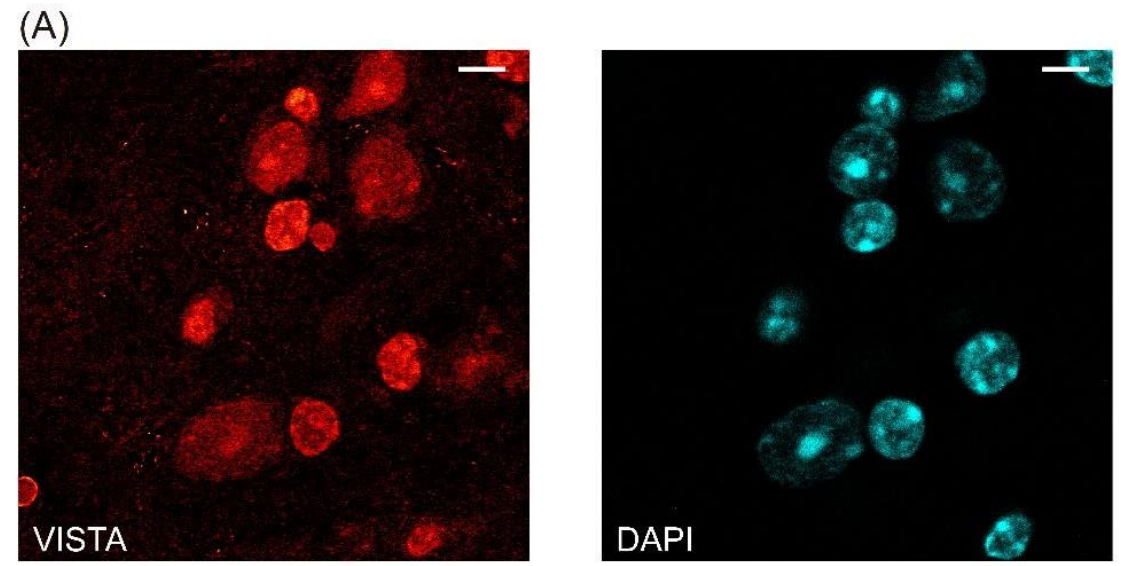

(B)
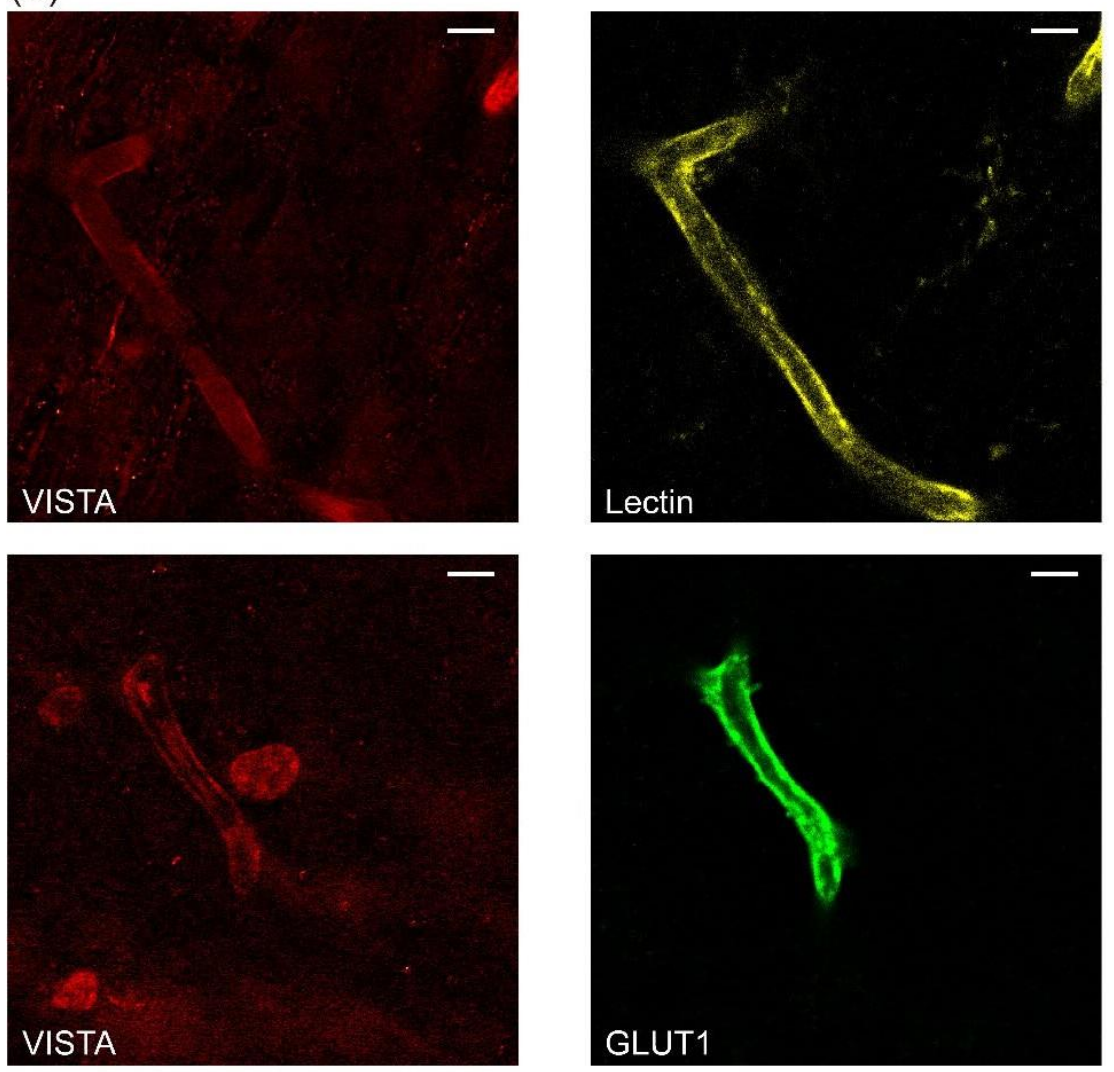

Fig. S4 VISTA images (left column) with the corresponding fluorescence stains (right column) for (A) cell nuclei and (B) blood vessels (fluorescence confirmations for blood vessels include both the lectin stains and the anti-GLUT1 antibody labeling). 
(A)

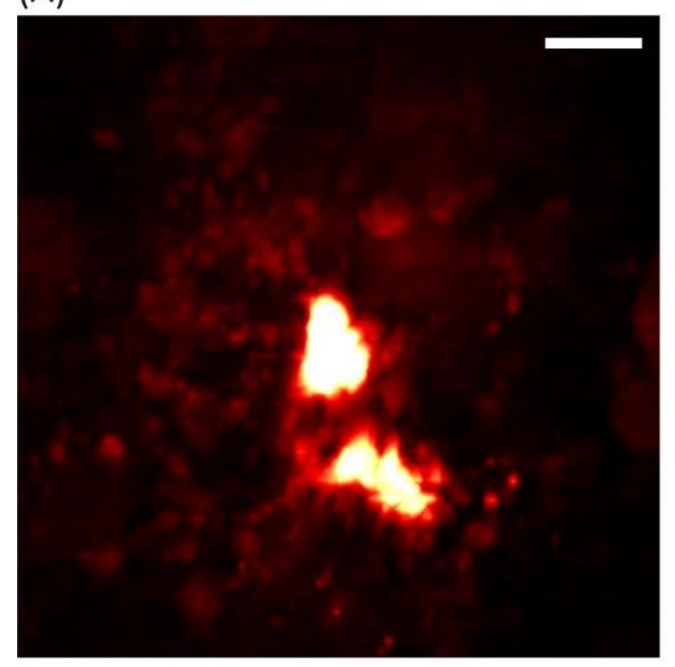

(B)

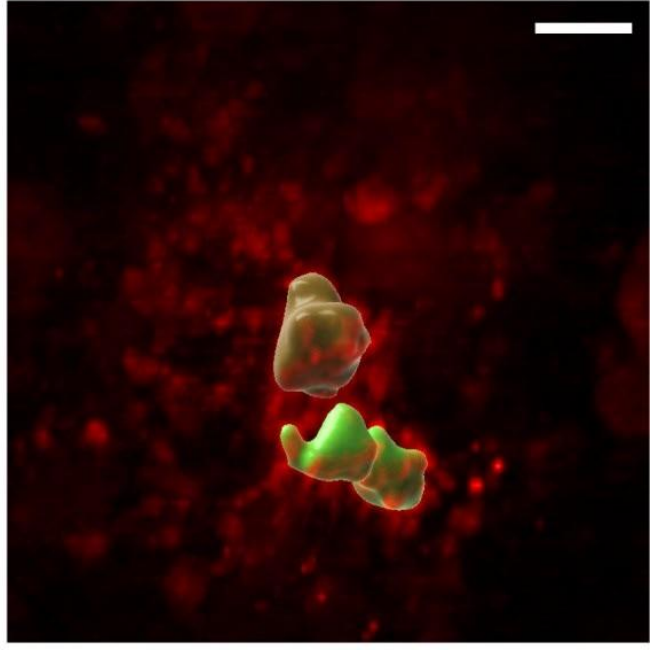

Fig. S5 (A) Intensity projection of the A $\beta$ plaques in 3D. (B) Surface rendering for (A) showing the core of the aggregate. Scale bars: $20 \mu \mathrm{m}$ 


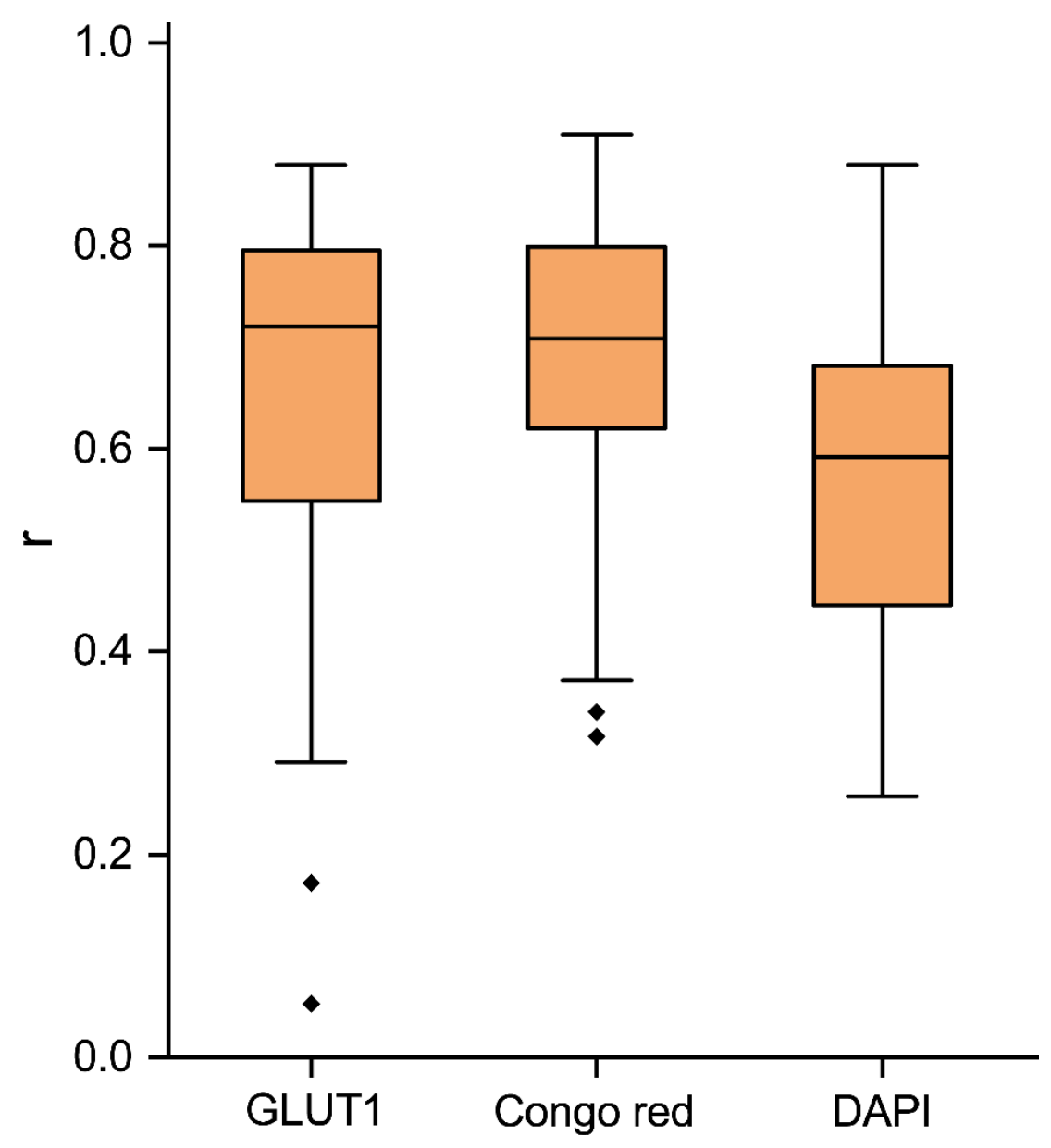

Fig. S6 Model performance of the U-Net models quantified by Pearson's correlation coefficient (r). The boxes indicate the 25th, 50th, and 75th percentile of the Pearson's $r$ for each model, with whiskers indicating 1.5 interquartile range. Glut1, N=47; Congo red, N=25; DAPI, N=138. 


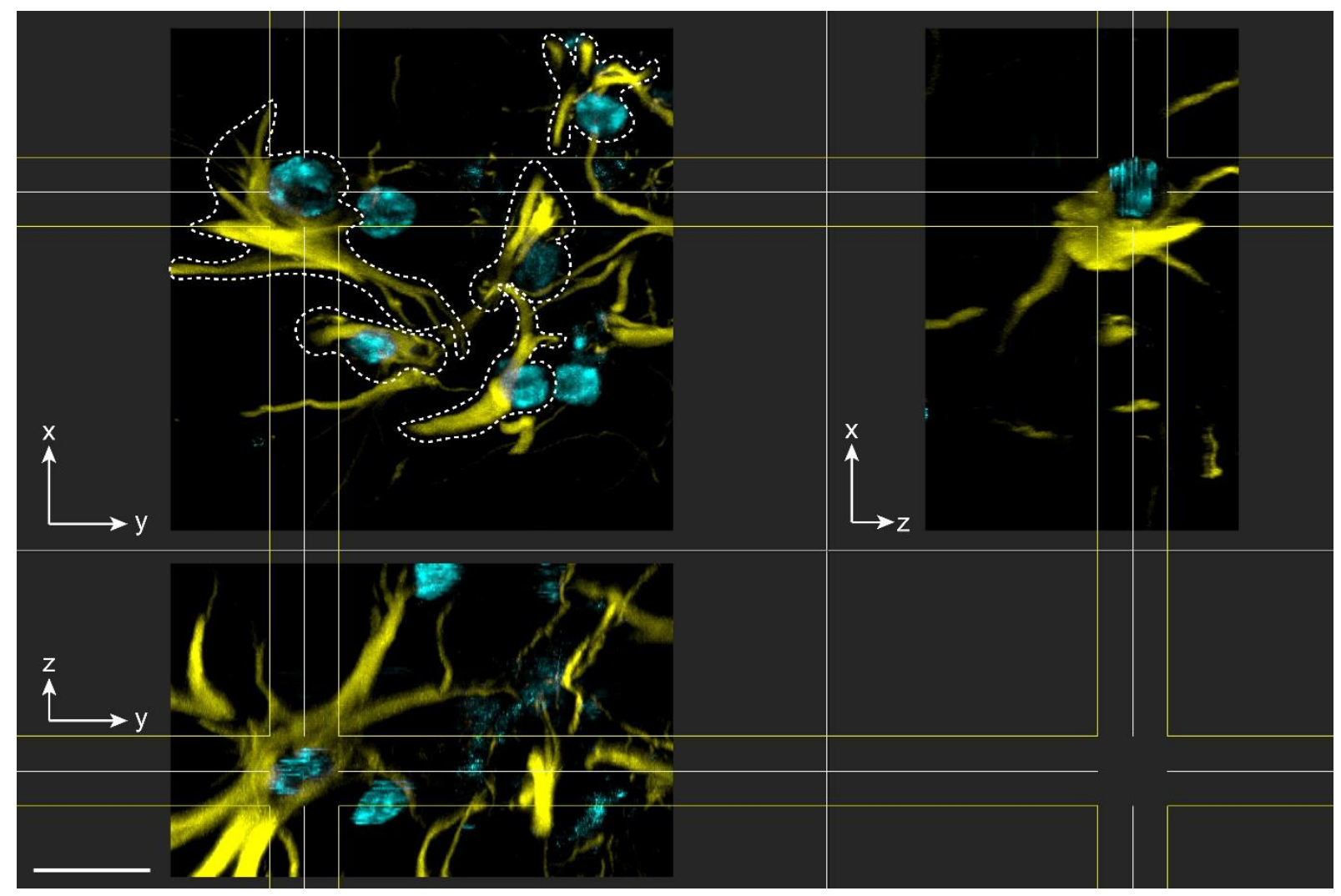

Fig. S7 Maximum intensity projection of predicted DAPI (v-DAPI, cyan) and fluorescencelabeled GFAP (yellow) for the cell set shown in the first row of Fig. 6 . The cells circled by dashed lines were identified as astrocytes based on the GFAP staining and the v-DAPI pattern. 

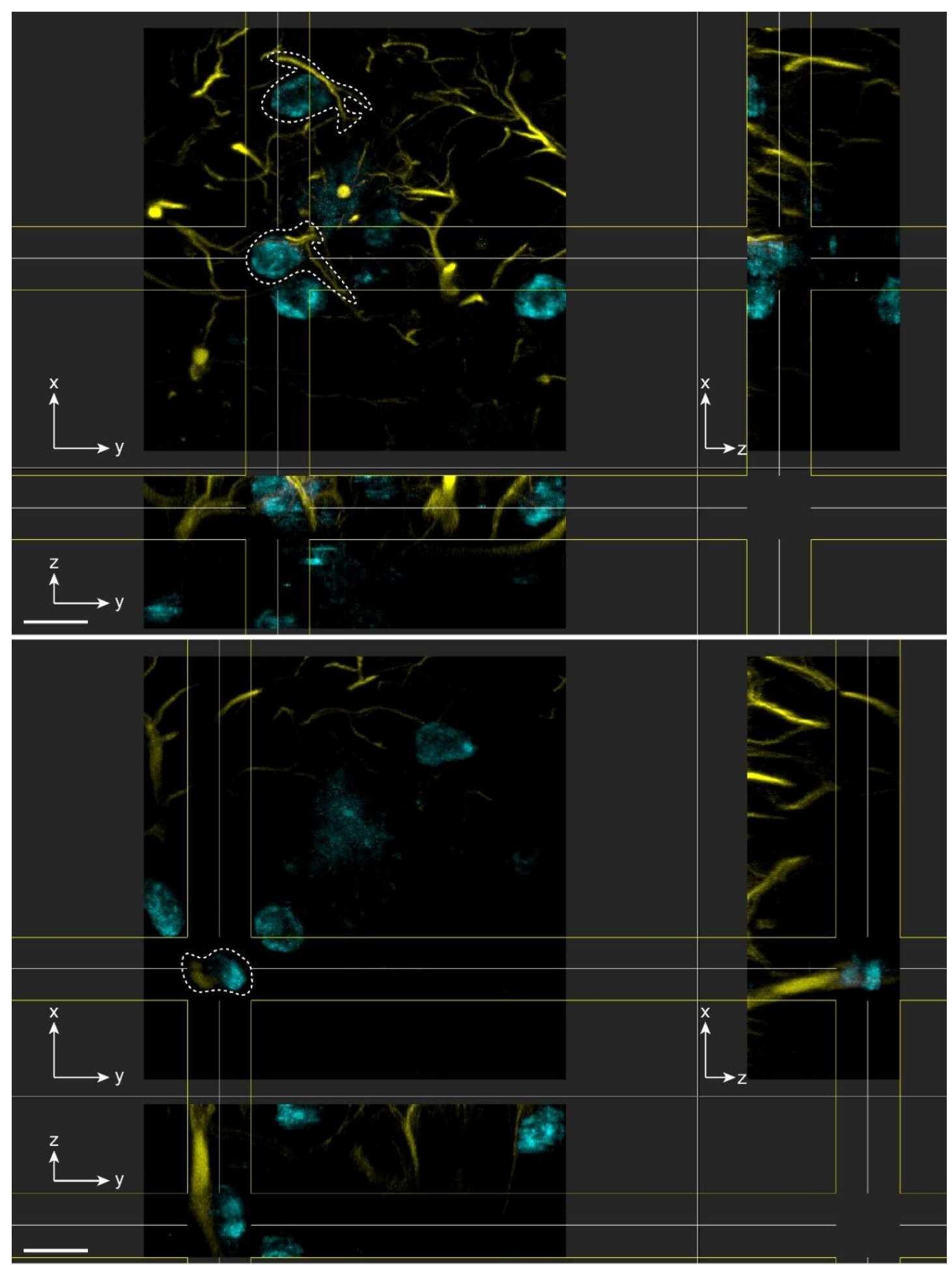

Fig. S8 Two maximum intensity projections of predicted DAPI (v-DAPI, cyan) and fluorescencelabeled GFAP (yellow) for the cell set shown in the second row of Fig. 6. The cells circled by dashed lines were identified as astrocytes based on the GFAP staining and the v-DAPI pattern. 
(A)

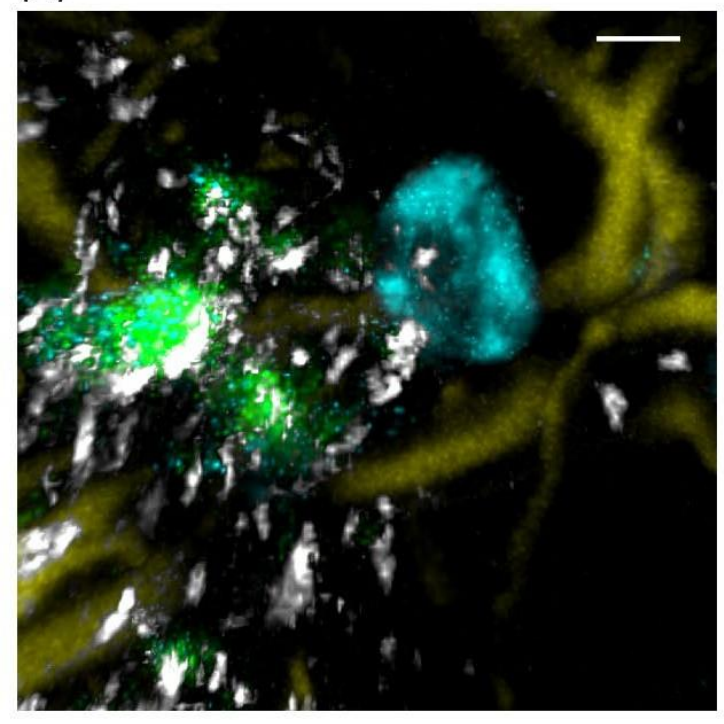

(B)

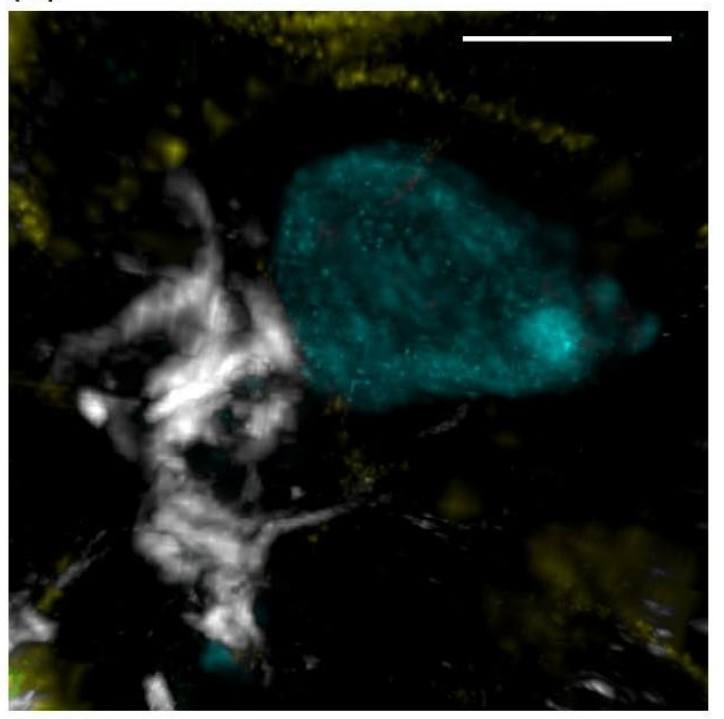

Fig. S9 Zoomed-in views of the dotted filament structure (A) and the ribbon-like structure (B) from the image set shown from the top row and the bottom row in Fig 6, respectively. Cyan: vDAPI. White: v-peripheral plaque. Yellow: GFAP. Green: v-Congo red. Scale bars: $10 \mu \mathrm{m}$. 